Service social

\title{
L'heure des bilans. Synthèse des débats tenus lors du colloque de juin 1999
}

\section{Guylaine Boucher}

Volume 47, numéro 1-2, 1998

Évaluation - Colloque 1999

URI : https://id.erudit.org/iderudit/706778ar

DOI : https://doi.org/10.7202/706778ar

Aller au sommaire du numéro

Éditeur(s)

École de service social de l'Université Laval

ISSN

1708-1734 (numérique)

Découvrir la revue

Citer ce document

Boucher, G. (1998). L'heure des bilans. Synthèse des débats tenus lors du colloque de juin 1999. Service social, 47(1-2), 11-16.

https://doi.org/10.7202/706778ar d'utilisation que vous pouvez consulter en ligne.

https://apropos.erudit.org/fr/usagers/politique-dutilisation/ 


\section{L'heure des bilans ${ }^{1}$}

Guylaine BOUCHER

Décriée par les uns, encensée par les autres, l'évaluation des programmes, des services et des politiques a pris racine dans la foulée des années 1980 et du début des années 1990 dans un esprit d'analyse des performances. Compressions budgétaires aidant, elle a été littéralement portée aux nues par de nombreux gestionnaires du réseau de la santé et des services sociaux qui ont trouvé là un outil susceptible de les appuyer dans leurs décisions administratives. Aujourd'hui, plusieurs personnes mettent en doute sa capacité réelle à améliorer les services.

C'est d'ailleurs principalement à cette question que la Fédération québécoise des centres de réadaptation pour les personnes présentant une déficience intellectuelle (CRPDI) et le Conseil québécois de l'agrément ont tenté de répondre lors de leur colloque commun du 3 juin 1999 à Montréal. L'événement avait pour thème La contribution de l'évaluation à l'amélioration des services sociaux et de santé aux niveaux local, régional et provincial et reposait sur deux grandes interrogations, soit : l'impact de l'évaluation sur l'amélioration des services et l'arrimage entre les niveaux local, régional et provincial sur le plan de l'évaluation.

1. Synthèse des débats tenus lors du colloque du 3 juin 1999, à Longueuil, sur la contribution de l'évaluation des services à l'amélioration des services sociaux et de santé, aux niveaux local, régional et provincial. 
Le présent article résumera les visions exprimées en atelier par les participants au colloque de juin dernier et les principaux enjeux qui s'en dégagent.

\section{L'IMPACT DE L'ÉVALUATION}

\section{Sur le terrain...}

Dresser le bilan des différents efforts d'évaluation n'est pas une mince tâche. Sur le plan local, s'il faut en croire les participants au colloque, l'évaluation des programmes, services et politiques est encore au stade embryonnaire. Faute d'indicateurs elle est, selon eux, beaucoup moins systématique que sur le plan régional ou national. Par conséquent, de nombreux établissements déplorent ne pas pouvoir compter sur des feed-backs significatifs qui leur permettraient d'ajuster les services, programmes ou politiques mis en œuvre.

$\mathrm{Si}$, pour plusieurs, l'évaluation a souvent été sacrifiée sur le bûcher des compressions budgétaires, d'autres au contraire prétendent qu'elle existe depuis toujours sur le plan local. Certains dépeignent même des programmes relativement structurés d'évaluation de la satisfaction de la clientèle ou des employés réalisés à partir de sondages auprès des usagers, de leurs familles et des professionnels. II s'en trouve également pour dire que les comités d'usagers, les conseils de médecins dentistes, les conseils multidisciplinaires et les bilans annuels sont autant de structures et de mécanismes d'évaluation utilisés ou utilisables sur le plan local.

Enfin, de manière générale, tous affirment que l'évaluation ne peut pas avoir d'impact significatif au point de vue local sans un plan d'action et des objectifs à la fois précis et concrets. En réponse à cette exigence, plusieurs ont souligné le fait que l'on greffe de plus en plus de considérations qualitatives aux statistiques traditionnellement utilisées pour vérifier l'atteinte des objectifs. Depuis quelques années, dans un souci d'amélioration continue de la qualité, plus d'importance a été effectivement accordée aux liens existant entre les besoins des clients et les moyens mis en place pour les satisfaire, de même qu'aux besoins de formation des employés. Une façon de faire que les participants au colloque jugent positive et qui les rapproche, non seulement des indicateurs définis par la Fédération québécoise des centres de 
réadaptation pour les personnes présentant une déficience intellectuelle, mais surtout de la démarche d'agrément proprement dite.

Régionalement parlant, le tableau de l'évaluation est tout autre. En fait, tous ou presque s'entendent pour dire que l'évaluation y est très largement présente ou en pleine structuration. La révision périodique des plans régionaux d'organisation de services serait au cœur des démarches d'évaluation régionale.

Cependant, nombreux sont ceux qui déplorent le fait qu'elle soit axée sur l'état de l'implantation des services plutôt que sur leurs impacts. Résultat : il est très difficile de savoir si les programmes mis en place ont réellement permis l'amélioration de la qualité de vie des clients. D'autres regrettent que l'évaluation régionale soit effectuée sur la base de paramètres extérieurs aux établissements et le plus souvent définis par le ministère de la Santé et des Services sociaux. Une situation qui contribue à mettre en doute la fiabilité des constats obtenus au terme de l'évaluation, d'autant que l'information rendue publique à la suite des évaluations est très mince.

Sur le plan provincial, l'évaluation se définit essentiellement sous l'angle de la politique clientèle. De type qualitatif, les évaluations réalisées ont notamment permis de mettre en lumière les réels besoins de formation des intervenants psychosociaux. La question de l'évaluation des actions posées à l'égard des personnes âgées qui présentent une déficience intellectuelle a aussi été explorée. Certains déplorent le fait que les résultats obtenus par les grands mouvements d'évaluation provinciaux demeurent peu diffusés. Enfin, plusieurs confirment qu'ils fondent beaucoup d'espoir sur les systèmes d'informationclientèle et le Sommet 2000.

\section{AU POINT DE VUE DE L'UTILITÉ ET DE L'AMÉLIORATION DES SERVICES}

La seconde question à laquelle les participants au colloque ont été invités à répondre porte sur l'utilité que revêt l'évaluation aux divers paliers d'intervention. Vient ensuite la question de l'amélioration ou non des services suivant l'évaluation.

Sur le plan local, plusieurs ont souligné le fait que les exercices d'évaluation ont permis de constater le chemin parcouru et d'ajuster les 
services en réponse aux véritables besoins des clients. L'établissement d'outils de gestion efficaces et la motivation des employés figurent également au nombre des avantages qui découlent de l'évaluation. Pour d'autres, l'évaluation peut être d'une aide précieuse pour la prise de décision, à la condition qu'elle soit orientée vers les besoins des clients.

De nombreux participants au colloque ont affirmé que l'utilité et la portée de l'évaluation seraient beaucoup plus grandes si les établissements pouvaient compter sur des outils adéquats.

Des constats qui peuvent se transposer sur le plan régional, où les activités d'évaluation font très rarement appel aux clients ou aux établissements eux-mêmes. À ce sujet, l'examen du plan régional d'organisation des services a été mentionné comme un exemple parfait de la non-contribution de tous les acteurs concernés.

Par ailleurs, il s'en trouve également pour dire que l'évaluation au niveau régional permet véritablement de mesurer le niveau de compétence des divers intervenants et de leurs institutions. À une condition toutefois : celle de pouvoir compter sur une volonté politique ferme et véritable non seulement d'évaluer les résultats, mais aussi d'intégrer les changements qui s'imposent au processus de gestion. Le tout, en évitant de tomber dans le piège de la comparaison entre régions.

Quant à l'utilité de l'évaluation au niveau provincial, plusieurs affirment qu'elle contribue à la définition des orientations, et ce, malgré une certaine résistance au niveau national à l'égard de ce type de pratique. Une attitude que la crainte du changement et de la critique explique.

De là à dire que l'évaluation réalisée à tous les niveaux contribue à améliorer la qualité des services, les participants au colloque se sont montrés prudents. En fait, la majorité d'entre eux se disent convaincus qu'elle peut considérablement améliorer les services tant sur le plan local, régional que provincial, à la condition qu'elle ne se bute pas aux considérations politiques et qu'elle serve véritablement le client et non les visées budgétaires. Par ailleurs, plusieurs entretiennent beaucoup d'espoir sur la portée qu'agrément et inspection combinés pourraient avoir. 


\section{Satisfaisant ou non?}

Convaincues du bien-fondé de l'évaluation, les personnes réunies en ateliers ont cependant soulevé divers points sur lesquels il serait opportun d'agir pour une meilleure efficacité des processus d'évaluation. Une implication accrue des clients et des employés figure au nombre des éléments à améliorer, tout comme le développement de liens plus étroits entre les milieux de pratique et le monde universitaire. Par ailleurs, pour être pleinement satisfaisant, le processus d'évaluation devrait être intégré systématiquement à la gestion des activités et bénéficier d'un budget précis qui lui serait exclusivement réservé. Une plus grande cohérence entre les niveaux régional et provincial contribuerait aussi à rendre les exercices d'évaluation plus efficients. Enfin, à leur avis, rien de ce qui est fait en matière d'évaluation ne pourra être significatif sans une réelle volonté politique à tous les niveaux (local, régional et provincial).

\section{ARRIMAGE ENTRE LE LOCAL, LE RÉGIONAL ET LE PROVINCIAL}

Faire de l'évaluation est une chose. En faire de manière concertée en est une autre. Les participants au colloque ont été invités à se pencher sur la question de l'arrimage entre les divers niveaux d'intervention, soit le local, le régional et le provincial. Un arrimage qu'ils qualifient de largement insatisfaisant, voire carrément inexistant dans certains cas. C'est que leurs attentes vis-à-vis de la concertation sont très grandes. II est, à leur avis, absolument impératif que les trois paliers aient une volonté véritable de procéder par évaluation et qu'ils puissent compter sur une expertise et sur des outils efficaces. La mise en disponibilité systématique d'un budget d'évaluation pour chaque projet figure également dans la liste des éléments incontournables. Enfin, les participants ont aussi largement insisté sur l'importance que les divers paliers, y compris le local, s'entendent sur les objectifs évalués avant d'amorcer quelque processus que ce soit. L'utilisation de l'évaluation à des fins de contrôle est par ailleurs exclue.

Des attentes qui commandent des défis majeurs, dont celui de favoriser un échange d'expertise entre les divers paliers et d'apprendre à faire connaître les bons coups. Plus encore, il importe de faire en sorte que les décisions politiques pèsent moins lourd dans la balance 
afin que les évaluations soient, le plus possible, axées sur le client. Une autre façon de dire qu'un processus d'évaluation ne saurait avoir sa raison d'être si on ne s'assure pas d'abord que les décisions prises à la suite de cette évaluation correspondent véritablement aux intérêts du milieu concerné.

Mais encore faut-il savoir comment et par où commencer. Amenés à réfléchir à cette question, les participants au colloque ont identifié de multiples pistes de travail. La mise sur pied d'un système de données informationnelles figure au nombre de ces éléments. II en va également ainsi de la mise en place d'un site Internet où seraient répertoriés tous les outils d'évaluation actuellement disponibles. Pour d'autres, sur le plan local, le simple respect des mandats dévolus à chacun serait un excellent point de départ. Une façon de faire qui n'est pas très loin de celle voulant que tout processus d'évaluation doit reposer sur des mandats et des objectifs clairs, connus et partagés par tout le monde. Une approche qui nécessite la prise en compte, sur le plan provincial, des réalités propres au palier régional et de même pour le local. Enfin, placer la clientèle au centre de tout effort d'évaluation serait, à leur avis, assurément porteur de succès, tout comme faire de l'évaluation un choix organisationnel. Ultimement, pour contourner les résistances auxquelles ils peuvent se buter en cours d'évaluation, les participants ont rappelé l'importance de proposer des mesures incitatives aux personnes en cause, de la formation par exemple.

Guylaine BOUCHER Secrétaire du colloque 